\title{
An Analysis of Li Ion Secondary Battery Materials by Using Focused Ion Beam Micro-sampling Technique and Cold Field Emission Scanning Transmission Electron Microscope
}

\author{
T. Tanigaki,* K. Ito,* K. Nakamura,** Y. Nagakubo,** J. Azuma,** and T. Kanemura*
}

* Analytical Service Center, Hitachi High-Tech Manufacturing \& Service Corporation, 1040 Ichige, Hitachinaka, Ibaraki 312-0033, Japan

** Hitachi High-Technologies Corporation, 882 Ichige, Hitachinaka, Ibaraki 312-8504, Japan

In order to decrease green house gases, hybrid electric vehicle and electric vehicle have been developed. The using of Li ion secondary battery for hybrid electric vehicle or electric vehicle has increased in the world. The development of a high capacity and high power Li ion secondary battery is strongly demanded by motor companies. Understandings of mechanisms of charge, discharge and damage are very important for developing a new type Li ion secondary battery. Thus, there are needs of an analysis of Li distribution in an electrode of the battery. The experiments of the measurement of the Li distribution in the electrode before charge and after charge have been carried out.

The micro-sampling method using focused ion beam (FIB) (FB-2100, Hitachi High-Technologies Co.) [1] was used for preparing the thin film on the edge of Mo mesh for the microscopic analysis. First, the initial state of the material was analyzed using a cold field emission (Cold FE) scanning transmission electron microscope (STEM) with Cs corrector (HD-2700A, Hitachi HighTechnologies Co.). The accelerating voltage was $200 \mathrm{kV}$. Li cannot be analyzed by energy dispersive $\mathrm{X}$-ray spectroscopy. Therefore, Li was analyzed by using electron energy loss spectroscopy (EELS). Since, the anode materials often contain $\mathrm{Mn}, \mathrm{Fe}, \mathrm{Co}$ and $\mathrm{Ni}$, the EELS spectra of metal M edge and Li-K edge overlap with in $50 \sim 70 \mathrm{eV}$ range. This makes it difficult to measure the Li distribution by using EELS. Taking the advantage of Cold FE electron gun, high energy resolution EELS spectrum imaging (EELS-SI) [2,3] was acquired. The energy resolution was $0.35 \mathrm{eV}$ at zero loss. The $\mathrm{Li}$ distribution was obtained by analyzing each spectrum in SI. Second, the micro-sample was directly put in the experimental Li ion battery cell and charged. The sample was taken out from the cell and some adhesive materials were spattered by FIB. Finally, the sample state after the charge was analyzed. Li containing materials after inserted the Li ion secondary battery are very sensitive to air. Especially, the humidity is forbidden strictly. An atmosphere isolation type compatible FIB/STEM holder was used in this experiment. Fig. 1 shows the photographs of the isolation holder. Fig. 1 (a) and (b) show the open and close position.

The results of $\mathrm{LiMn}_{2} \mathrm{O}_{4}$ particle, which is one of the anode materials, are shown in Fig. 2. Fig.2 (a) and (b) show the high angular annular dark field (HAADF) STEM image and the Li distribution before the charge. Fig. 2 (c) and (d) show those after the charge. By charging, the Li distribution became totally low and there were residual Li partially. It was found that the loss of Li mainly took place at the surface area of the particles.

Using the FIB micro-sampling technique, the atmosphere isolation holder, Cold FE-STEM and EELS-SI method, the measurement of the Li distribution in the electrode before the charge and after the charge have been achieved. 
References

[1] T. Ohnishi et al., Proc. $25^{\text {th }}$ Int. Symp. Testing and Failure Analysis (1999) 449.

[2] C. Jeanguillame et al., Ultramicroscopy 28 (1989) 252.

[3] J. M. Titchmarsh et al., J. Microscopy 184 (1996) 195.

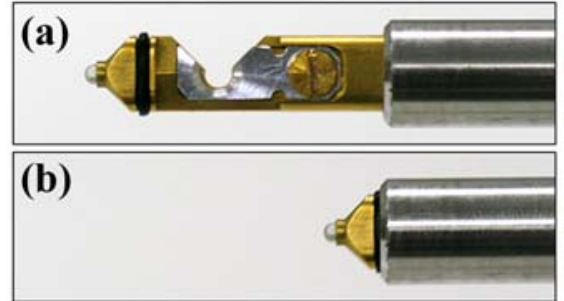

(c)

Fig. 1. The photographs of the atmosphere isolation type compatible FIB/STEM holder. (a) FIB and STEM position of the holder tip. (b) Atmosphere isolating position during the transportation of the sample between FIB and STEM. (c) The appearance of the holder.

(a)

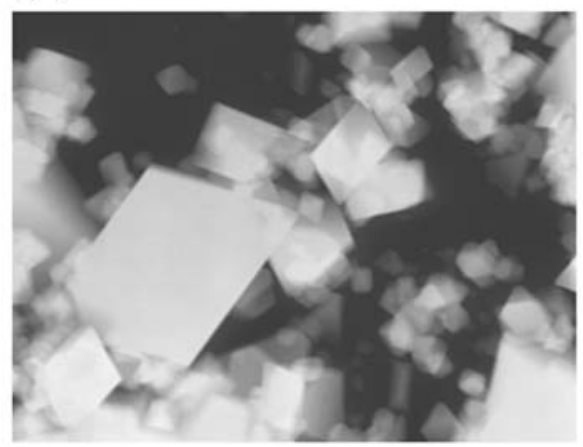

(c)

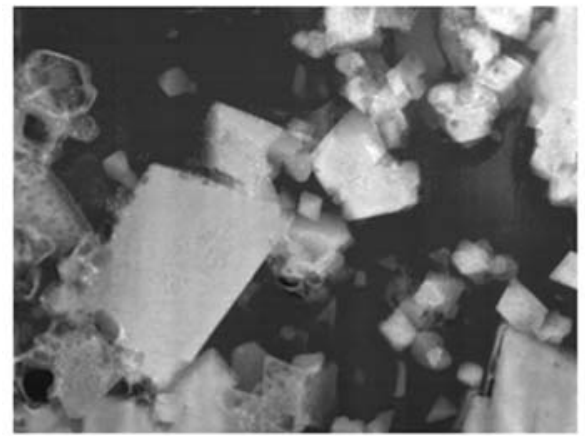

(b)

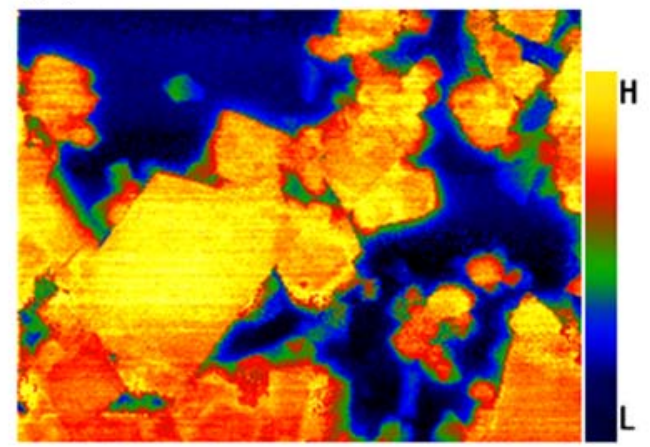

(d)

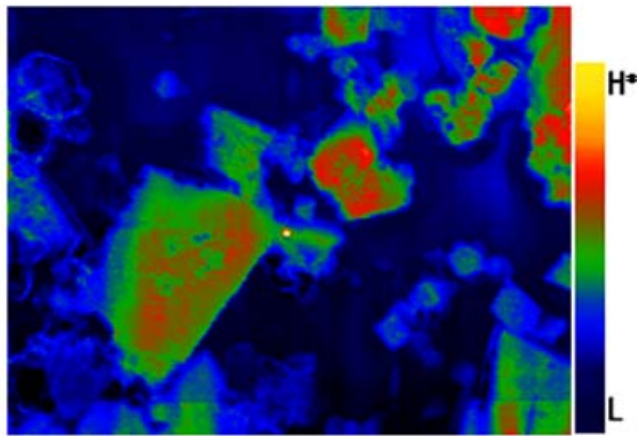

$400 \mathrm{~nm}$

Fig. 2. (a) and (b) show the HAADF STEM image and the Li distribution before the charge. (c) and (d) show those after the charge. 